\title{
Collaborative care for depression in UK primary care: a randomized controlled trial
}

\author{
D. A. Richards ${ }^{1 *}$, K. Lovel1 ${ }^{2}$, S. Gilbody ${ }^{1}$, L. Gask ${ }^{3}$, D. Torgerson ${ }^{1}$, M. Barkham ${ }^{4}$, M. Bland ${ }^{1}$, P. Bower ${ }^{3}$, \\ A. J. Lankshear ${ }^{5}$, A. Simpson ${ }^{1}$, J. Fletcher ${ }^{2}$, D. Escott ${ }^{2}$, S. Hennessy ${ }^{1}$ and R. Richardson ${ }^{1}$ \\ ${ }^{1}$ Department of Health Sciences, University of York, UK \\ ${ }^{2}$ Department of Nursing, Midwifery and Social Work, University of Manchester, UK \\ ${ }^{3}$ National Primary Care Research and Development Centre, University of Manchester, UK \\ ${ }^{4}$ Centre for Psychotherapy Services Research, University of Sheffield, UK \\ ${ }^{5}$ School of Nursing and Midwifery Studies, University of Cardiff, UK
}

Background. Collaborative care is an effective intervention for depression which includes both organizational and patient-level intervention components. The effect in the UK is unknown, as is whether cluster- or patient-randomization would be the most appropriate design for a Phase III clinical trial.

Method. We undertook a Phase II patient-level randomized controlled trial in primary care, nested within a clusterrandomized trial. Depressed participants were randomized to 'collaborative care' - case manager-coordinated medication support and brief psychological treatment, enhanced specialist and GP communication - or a usual care control. The primary outcome was symptoms of depression (PHQ-9).

Results. We recruited 114 participants, 41 to the intervention group, 38 to the patient-randomized control group and 35 to the cluster-randomized control group. For the intervention compared to the cluster control the PHQ-9 effect size was 0.63 (95\% CI 0.18-1.07). There was evidence of substantial contamination between intervention and patient-randomized control participants with less difference between the intervention group and patient-randomized control group $(-2.99$, $95 \% \mathrm{CI}-7.56$ to $1.58, p=0.186)$ than between the intervention and cluster-randomized control group $(-4.64,95 \% \mathrm{CI}$ -7.93 to $-1.35, p=0.008)$. The intra-class correlation coefficient for our primary outcome was $0.06(95 \%$ CI $0.00-0.32)$.

Conclusions. Collaborative care is a potentially powerful organizational intervention for improving depression treatment in UK primary care, the effect of which is probably partly mediated through the organizational aspects of the intervention. A large Phase III cluster-randomized trial is required to provide the most methodologically accurate test of these initial encouraging findings.

Received 5 February 2007; Revised 5 June 2007; Accepted 22 June 2007; First published online 6 September 2007

Key words: Collaborative care, complex intervention, depression, primary care, randomized controlled trial.

\section{Introduction}

Depression is a major health problem causing substantial disability and set to become the second largest cause of global disability by 2020 (WHO, 2001). In the UK depression and anxiety are estimated to cost the economy $£ 17$ bn in lost output with a $£ 9$ bn impact on the Exchequer through benefit payments and lost tax receipts (Layard, 2006). Only one in four depressed people receive effective pharmacological treatment and less than $10 \%$ a talking therapy (Singleton et al. 2001). Bower \& Gilbody (2005) have identified four types of organizational strategies to improve this

\footnotetext{
* Address for correspondence: Professor D. A. Richards, Department of Health Sciences, Seebohm Rowntree Building, University of York, University Road, Heslington, York, North Yorkshire, United Kingdom.

(Email: dr17@york.ac.uk)
}

situation: training and the use of guidelines for general practitioners and primary care staff; ' consultation liaison', whereby specialist mental health practitioners advise on the care of individual patients in primary care; 'collaborative care', an enhanced form of consultation liaison which also includes a case manager to deliver care and liaise between GP, specialist and patient; and 'replacement referral' which refers to the deployment of specialists in secondary or primary care to whom GPs can refer.

A systematic review (Gilbody et al. 2003) of 36 such organizational intervention studies concluded that effective strategies require complex interventions at the 'systems level', consisting of: (1) a multi-professional approach to patient care; (2) a structured patient management plan; (3) scheduled patient follow-ups; and (4) enhanced inter-professional communication (Wagner et al. 1996; Gunn et al. 2006). The most 
effective systems-level intervention in this review was 'collaborative care' (Von Korff \& Goldberg, 2001; Simon, 2006).

Although collaborative care improves outcomes over usual care (Katon et al. 1999; Wells et al. 2000; Unutzer et al. 2002), two recent systematic reviews found small to medium mean effect sizes of either 0.24 (95\% CI 0.17-0.32) (Gilbody et al. 2006) or 0.40 (95\% CI 0.20-0.60) (Gensichen et al. 2005). The effects associated with individual studies varied significantly, reflecting variation in the content of these 'complex' interventions (MRC, 2000). Further, most of the studies originated from the USA (Gilbody et al. 2006).

Although there have been calls for the implementation of collaborative care in the UK (Simon, 2006), these have not been supported by UK clinical guidelines (National Institute for Health and Clinical Excellence, 2004) and may be premature given that it is not known exactly which models of collaborative care work best, and whether the model will generalize to the UK. In other areas of mental health such as assertive community treatment (Killaspy et al. 2005), the adoption of complex interventions based on international/US data before UK evaluation has resulted in ineffective UK service developments.

We adopted the phased approach (Campbell et al. 2000) recommended by the Medical Research Council (MRC) for investigating complex interventions (MRC, 2000). We developed a UK-specific collaborative care intervention for depression, based on analysis of 'active ingredients' in published interventions (Gilbody et al. 2006) and in-depth qualitative research with stakeholders (Richards et al. 2006a). We then tested it in an exploratory Phase II randomized controlled trial.

Cluster-randomized controlled trials are recommended for testing systems-level interventions such as collaborative care (Ukoumunne et al. 1999), since patient-randomized trials may be vulnerable to contamination. Patients in the control group may be influenced by system-level changes such as advice from specialists and changes to the process of care. Contamination in a patient-randomized trial may result in underestimating the real effect size of collaborative care. However, cluster-randomized trials require larger patient samples and often greater resources. Our Phase II trial, therefore, used an unusual design, nesting patient-level randomization within a cluster-randomized controlled trial to investigate the presence and magnitude of contamination (Fig. 1). We report the results of that randomized controlled trial here, the first UK trial of collaborative care and an early test of the utility of the MRC's complex-interventions framework.
Ethical approval was given by the South West Multi-site Research Ethics Committee.

\section{Objectives}

The objectives were:

(1) To estimate an effect size for a UK-specific collaborative care protocol.

(2) To determine whether cluster or patient randomization would be the most appropriate design for a Phase III trial.

\section{Method}

General practice sites were randomly allocated to treatment or cluster control conditions from four primary-care trusts (PCT) in the northern UK, stratified by PCT. Almost all practices had a deprivation index higher than the UK national average and a number were from areas where black and minority ethnic groups were strongly represented. Patients in the treatment cluster group were then individually randomized to either collaborative care or usual care control. Allocation was by a remote computergenerated number sequence concealed from researchers and conducted independently after patients were enrolled in the study by research interviewers. The randomization team at the trials unit informed patients, GPs and, where appropriate, case managers, of participant allocation. This created three study groups (cluster-randomized controls, individually randomized intervention patients, and individually randomized control patients). Fig. 2 details the consort diagram. To try to reduce the possibility of recruitment bias GPs were given no information about the allocation of their practice.

\section{Sample}

We recruited patients from primary care aged $>18$ years diagnosed as depressed by a GP, confirmed by a score of $\geqslant 5$ on the depression section of the Standard Clinical Interview for DSM-IV (SCID; Spitzer et al. 1992) undertaken by trained research assistants. We excluded patients with post-natal, bereavement or physical causes for their depression. We only included patients with a newly identified episode of major depression, defined as a current episode of GP-initiated treatment of not more than 1 month's duration. We excluded patients reporting active suicidal plans and those with a primary drug or alcohol dependence. Although a standard power calculation based on detecting treatment effects would be the conventional approach to determining the sample size for a clinical trial, our Phase II study was designed to help inform 
The collaborative care intervention for depression consists of two aspects:

- A component $\mathbf{A}$ that impacts on the individual patient (e.g. medication management from the case manager).

- A component B that impacts on the general practice (e.g. feedback of patient information to the GP).

In a standard, individually randomized trial, component $\mathbf{A}$ only influences patients who are randomly allocated to receive it, whereas component $\mathbf{B}$ may potentially influence all patients who are under the care of a particular practice. Contamination occurs if the outcome of patients randomized to the control group is influenced by $\mathbf{B}$, even though those patients receive no benefit from $\mathbf{A}$.

In the design adopted in this study, a patient level randomization study is nested within a cluster level randomized study.

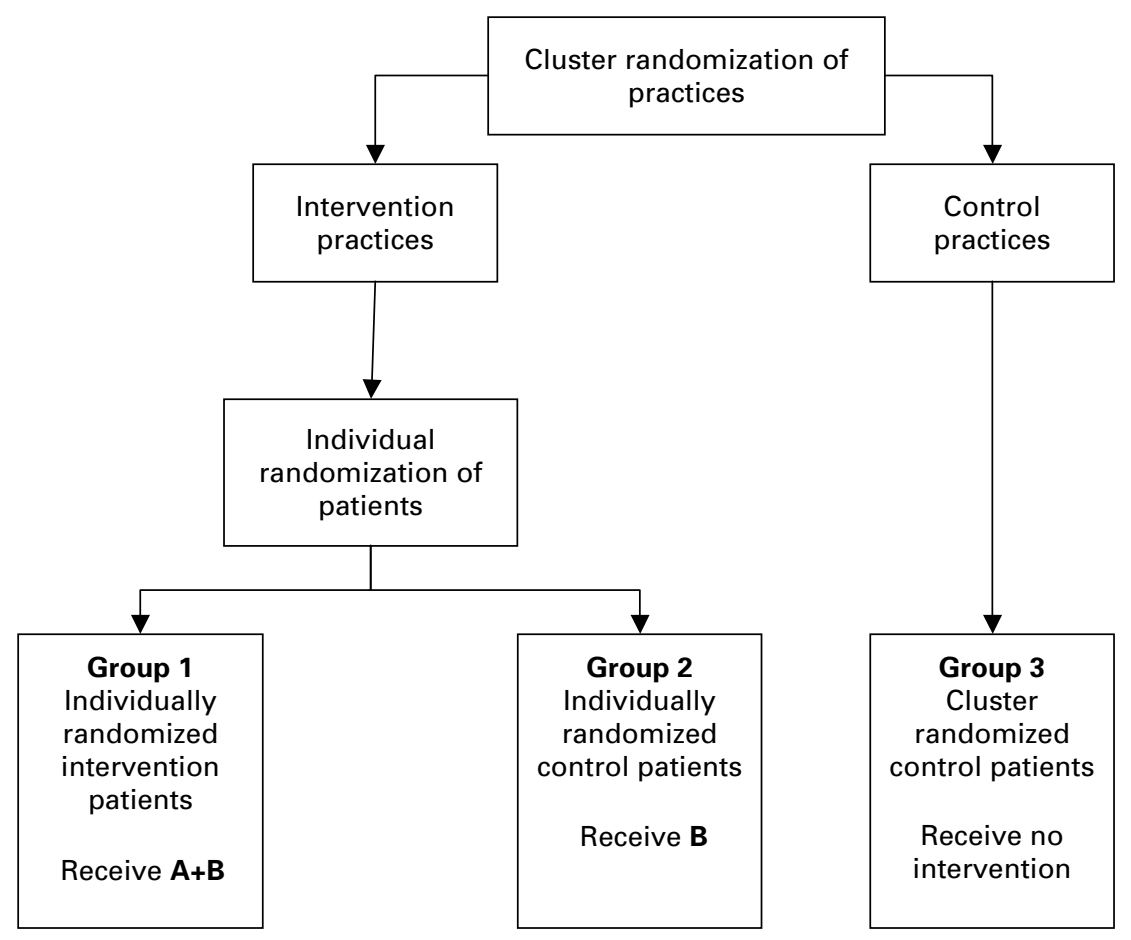

This has the advantage of allowing two analyses:

- The analysis of Group 1 versus Group 3 compares individually randomized intervention patients (who have received the individual patient component $\mathbf{A}$ and the practice component $\mathbf{B}$ ) with cluster-randomized control patients (who have received neither $\mathbf{A}$ nor $\mathbf{B}$ ). This analysis therefore provides the best overall indication of the effect of the entire collaborative care intervention (i.e. $\mathbf{A}+\mathbf{B}$ ).

- The analysis of Group 2 versus Group 3 compares individually randomized control patients (who have received the practice component B) with cluster-randomized control patients (who have received neither $\mathbf{A}$ nor B). This analysis therefore provides the best overall indication of the effect of the practice component (i.e. B). As noted above, this component $\mathbf{B}$ represents potential contamination in an individually randomised trial comparing Group 1 and Group 2.

Fig. 1. Investigating contamination in a trial of a complex organizational intervention.

the overall power calculation for a definitive Phase III trial to be used together with estimates of the 'expected treatment effect' (MRC, 2000) from published studies to allow us to estimate a plausible effect size for use in the sample size calculation for the definitive Phase III trial. Recruiting 32 patients in each of the patientrandomized groups within the treatment cluster would have given us a $95 \%$ confidence interval width of half an effect size, allowing us to test for an effect of 0.25 in either direction. Therefore, we aimed to recruit 


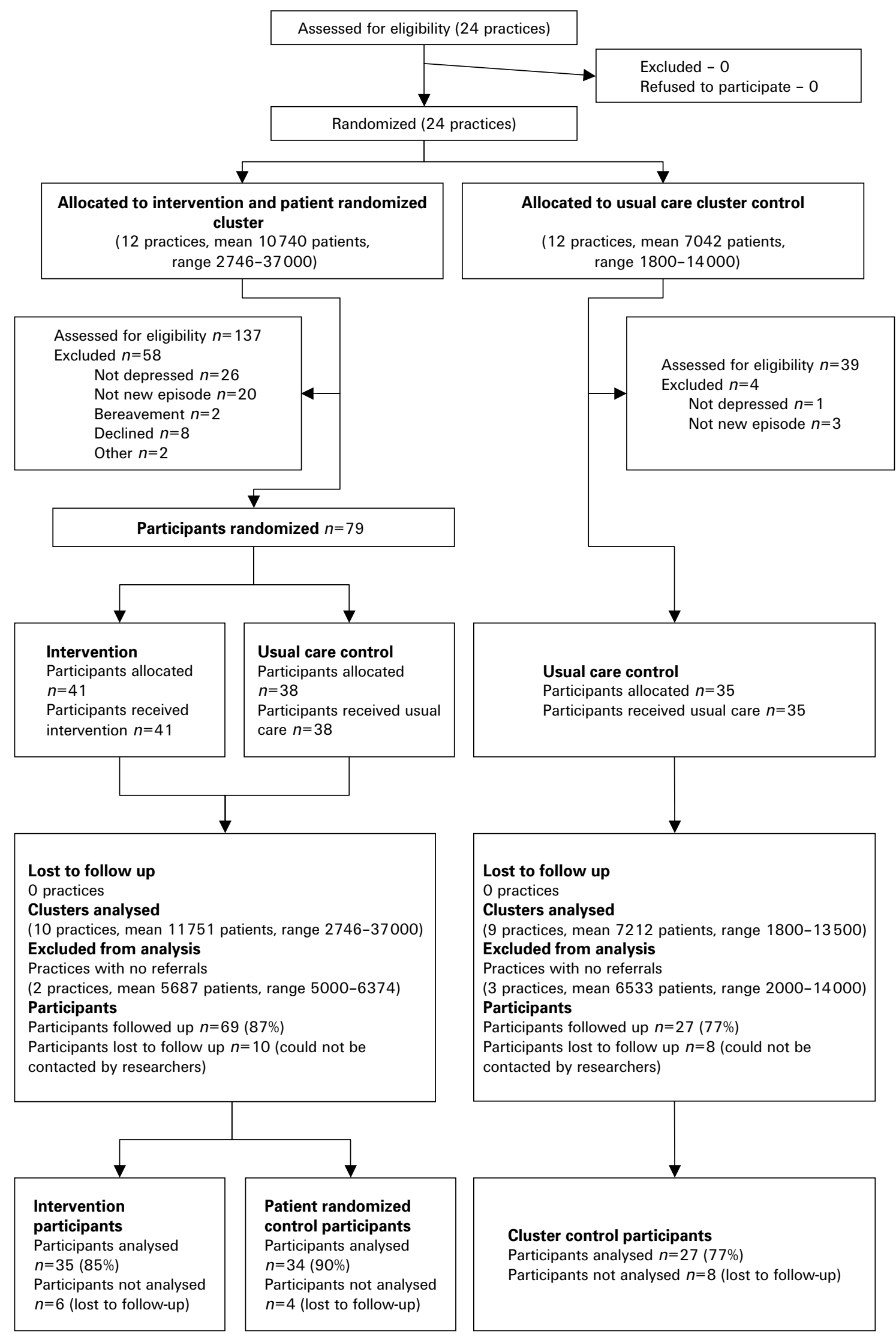

Fig. 2. Consort diagram. 
144 patients in total between the intervention and control clusters, 32 of the extra patients compensating for the design effect, the remainder to account for any attrition.

\section{Intervention}

Experimental

We developed a UK-specific collaborative care protocol in the modelling phase of our complexinterventions trial (Gilbody et al. 2006; Richards et al. 2006a), which met the four criteria for an organizational, systems-level intervention (Wagner et al. 1996; Gunn et al. 2006): (1) a multi-professional approach to patient care provided by a case manager working with the GP under weekly telephone supervision from specialist mental health medical and psychological therapies clinicians; (2) a structured management plan of medication support and behavioural activation - a structured cognitive-behaviourally based, depressionspecific psychological intervention which has equivalent efficacy to other more complex CBT interventions (Dimidjian et al. 2006; Cuijpers et al. 2007) but is simpler to use and thus more suitable for collaborative care (Jacobson et al. 1996; Martell et al. 2001). No other interventions were permitted for the duration of the trial; (3) scheduled patient follow-ups via a maximum of ten scheduled contacts over a period of 3 months, predominantly using the telephone; (4) enhanced inter-professional communication patientspecific written feedback to GPs via electronic records and personal contact. Case managers were a mix of professionals (nurse, counsellor and occupational therapist) and para-professionals (graduate primarycare mental health workers) all of whom received 2 days of protocol-specific training in addition to their existing clinical training and $30-45 \mathrm{~min}$ of supervision per week for the duration of the trial.

\section{Control}

Usual care management of depression by patients' GPs, including access to secondary services, and to best practice guidance published in local NHS depression protocols in the trial localities.

\section{Outcome measures}

The primary outcome was symptoms of depression as measured by the Patient Health Questionnaire-9 (PHQ-9; Kroenke et al. 2001). Secondary outcomes were the Clinical Outcomes in Routine Evaluation - Outcome Measure (CORE-OM; Barkham et al. 2001), measuring general wellbeing; the Short Form Health Survey (SF-36 v.2; Ware et al. 2000), measuring healthrelated quality of life. All assessments were completed at baseline and 3 months post-randomization by trained assessors blind to participant allocation.

\section{Analysis}

We aimed to determine a point estimate of the effect size of collaborative care, specific to the UK primarycare setting. We conducted analysis of covariance accounting for baseline imbalances in depression scores and clustering within the units of randomization using the Huber-White sandwich estimator (White, 1980) within Stata 8 (Stata Corp., College Station, TX, USA). We used an intention-to-treat approach to examine the mean differences between the three groups and the associated confidence intervals and calculated coefficients to represent the difference between the clusterrandomized controls and the intervention group in follow-up outcome measures. For the main analysis of the effectiveness of the intervention, we calculated the standardized effect size (mean difference divided by the pooled standard deviation) between the intervention and cluster-randomized control groups. We examined the degree of contamination by comparing the coefficients of individually randomized and cluster-randomized control groups. We examined clustering of outcomes within practices by calculating the intra-class correlation coefficient (ICC).

\section{Results}

We recruited 114 patients, 41 to the intervention group, 38 to the patient randomized control group and 35 to the cluster-randomized control group (Fig. 2) from February 2005 until March 2006. Table 1 details the sample characteristics. The average number of case manager/patient contacts was 6.46 (s.D. $=1.69$ ), taking a mean time per patient of $191.13 \mathrm{~min}$ (S.D. $=70.68$ ).

We found an effect size on PHQ-9 depression symptoms of 0.63 (95\% CI $0.18-1.07)$ for the intervention compared to the cluster control (Table 2). We found the intervention to be more effective than the cluster control on the CORE-OM $(0.45,95 \% \mathrm{CI}$ 0.11-1.01) and the mental component score of the SF-36 (0.67, 95\% CI 0.19-1.16) but not more effective on the physical component score of the SF-36 (0.11, $95 \%$ CI -0.49 to 0.72 ). No adverse events were reported in any group.

Evidence for substantial contamination was observed, as there was less difference in PHQ-9 depression outcomes between the intervention group and patient-randomized control group $(-2.99,95 \% \mathrm{CI}$ -7.56 to $1.58, p=0.186$ ) than between the intervention group and cluster-randomized controls (coefficient $-4.64,95 \%$ CI -7.93 to $-1.35, p=0.008)$. The ICC for our primary outcome was 0.06 (95\% CI 0.00-0.32). 
Table 1. Baseline characteristics of sample

\begin{tabular}{|c|c|c|c|c|}
\hline & $\begin{array}{l}\text { Intervention } \\
(n=41)\end{array}$ & $\begin{array}{l}\text { Patient-randomized } \\
\text { control }(n=38)\end{array}$ & $\begin{array}{l}\text { Cluster-randomized } \\
\text { control }(n=35)\end{array}$ & $\begin{array}{l}\text { Total } \\
(n=114)\end{array}$ \\
\hline \multicolumn{5}{|l|}{ Gender } \\
\hline Female & $32(78 \%)$ & $29(76 \%)$ & $27(77 \%)$ & $88(77 \%)$ \\
\hline Male & $9(22 \%)$ & $9(24 \%)$ & $8(23 \%)$ & $26(23 \%)$ \\
\hline \multicolumn{5}{|l|}{ Age (yr) } \\
\hline Mean (s.D.) & $42.63(12.33)$ & $43.00(14.88)$ & 41.71 (13.99) & 42.47 (13.62) \\
\hline Range & $20-72$ & $18-77$ & $19-71$ & $18-77$ \\
\hline \multicolumn{5}{|l|}{ Ethnic origin } \\
\hline White British & $38(93 \%)$ & $33(87 \%)$ & $27(77 \%)$ & $98(86 \%)$ \\
\hline Other & $3(7 \%)$ & $5(13 \%)$ & $8(23 \%)$ & $16(14 \%)$ \\
\hline \multicolumn{5}{|l|}{ Education } \\
\hline None & $9(22 \%)$ & $9(24 \%)$ & $10(29 \%)$ & $28(25 \%)$ \\
\hline GCSE/0 Level & $11(27 \%)$ & $9(24 \%)$ & $13(37 \%)$ & $33(29 \%)$ \\
\hline Post-GCSE/0 level & $16(39 \%)$ & $15(39 \%)$ & $9(26 \%)$ & $40(35 \%)$ \\
\hline Degree or higher & $3(7 \%)$ & $4(11 \%)$ & $3(9 \%)$ & $10(9 \%)$ \\
\hline Other or 'don't know' & $2(5 \%)$ & $1(3 \%)$ & 0 & $3(3 \%)$ \\
\hline \multicolumn{5}{|l|}{ Employment status } \\
\hline Employed/self-employed & $24(59 \%)$ & $14(40 \%)$ & $17(49 \%)$ & $56(49 \%)$ \\
\hline Not working & $17(41 \%)$ & $24(60 \%)$ & $18(51 \%)$ & $58(51 \%)$ \\
\hline \multicolumn{5}{|l|}{ Marital status } \\
\hline Married/cohabiting & $26(63 \%)$ & $25(66 \%)$ & $10(29 \%)$ & $61(54 \%)$ \\
\hline \multicolumn{5}{|l|}{ Baseline score means (s.D.) } \\
\hline PHQ-9 & $17.51(4.90)$ & $16.29(4.47)$ & $18.17(5.58)$ & $17.31(5.00)$ \\
\hline SCL-20 & $47.34(12.15)$ & $43.84(12.38)$ & $47.85(14.60)$ & $46.34(13.02)$ \\
\hline CORE-OM & $2.02(0.58)$ & $1.88(0.61)$ & $2.12(0.55)$ & $2.00(0.58)$ \\
\hline SF-36 MCS & $19.06(11.42$ & 20.33 (10.19) & $18.64(10.98)$ & $19.36(10.80)$ \\
\hline SF-36 PCS & $50.80(10.88)$ & $50.99(11.05)$ & $49.2(14.18)$ & $50.38(11.94)$ \\
\hline EQ5D & $0.55(0.26)$ & $0.56(0.30)$ & $0.43(0.32)$ & $0.52(0.29)$ \\
\hline
\end{tabular}

PHQ-9, Patient Health Questionnaire-9; SCL-20, Hopkins Symptoms Checklist Depression Scale; CORE-OM, Clinical Outcomes in Routine Evaluation - Outcome Measure; SF-36 MCS, Short Form Health Survey mental component score; SF-36 PCS, Short Form Health Survey physical component score; EQ5D, EuroQuol 5 Dimension Scale.

\section{Discussion}

We found a moderate to large effect (Cohen, 1988; Lipsey \& Wilson, 2001) of collaborative care, an effect which would be considered clinically significant under the guidelines for depression produced by the National Institute for Health and Clinical Excellence (2004), the first time this has been demonstrated in the UK. This effect is greater than that determined by systematic reviews (Gensichen et al. 2005; Gilbody et al. 2006) and equates to a mean difference between treated and usual care patients of 5 points on the PHQ9. Five points is the difference between symptoms of mild or moderate/severe intensity and between symptoms of moderate/severe and severe intensity. Furthermore, change in PHQ-9 scores achieved by the intervention patients from baseline to follow-up equates to a clinical shift of almost two categories of depression severity.
The optimal design for a full Phase III trial has also been clarified by the phased approach. We detected clear evidence of contamination, which has not always been accounted for in previous collaborative care studies (Gilbody et al. 2003). The individually randomized controls were closer to the intervention patients than to the cluster-randomized controls. Although striking, the precise mechanism of the contamination is unclear, but may relate to the sharing of information between case manager and GP. Examination of this mechanism may prove fruitful for the further development of interventions. As in any cluster-randomized trial, however, unmeasured differences between doctors could also explain some differences in outcomes, although we were careful to stratify cluster randomization by our four clinical sites to protect against this source of bias.

The main limitation of this study is the relatively small numbers in what was a Phase II trial. 
Table 2. Follow-up scores, coefficients of difference between intervention and patient-randomized control group with cluster controls, $95 \% \mathrm{CI}$ and $p$ values of the difference

\begin{tabular}{|c|c|c|c|}
\hline & Intervention & $\begin{array}{l}\text { Patient-randomized } \\
\text { control }\end{array}$ & $\begin{array}{l}\text { Cluster-randomized } \\
\text { control }\end{array}$ \\
\hline PHQ-9 & $n=35$ & $n=34$ & $n=27$ \\
\hline Mean (s.D.) & $8.80(7.02)$ & $10.27(7.51)$ & $13.82(8.32)$ \\
\hline Coefficient & -4.64 & -2.99 & N.A. \\
\hline $95 \%$ CI & -7.93 to -1.35 & -7.56 to 1.58 & \\
\hline$p$ value & 0.008 & 0.186 & \\
\hline CORE-OM & $n=39$ & $n=38$ & $n=32$ \\
\hline Mean (S.D.) & $2.02(0.58)$ & $1.88(0.61)$ & $2.12(0.55)$ \\
\hline Coefficient & 0.34 & -0.24 & N.A. \\
\hline $95 \%$ CI & -0.76 to 0.08 & -0.69 to 0.20 & \\
\hline$p$ value & 0.109 & 0.268 & \\
\hline SF-36 MCS & $n=39$ & $n=37$ & $n=33$ \\
\hline Mean (S.D.) & $19.06(11.42)$ & $20.33(10.19)$ & $18.64(10.98)$ \\
\hline Coefficient & 9.89 & 2.95 & N.A. \\
\hline $95 \%$ CI & 2.79 to 17.00 & -6.00 to 11.92 & \\
\hline$p$ value & 0.009 & 0.497 & \\
\hline SF-36 PCS & $n=39$ & $n=37$ & $n=33$ \\
\hline Mean (S.D.) & $50.80(10.88)$ & 50.99 (11.05) & $49.2(14.18)$ \\
\hline Coefficient & 0.91 & 1.49 & N.A. \\
\hline $95 \%$ CI & -3.89 to 5.72 & -3.02 to 6.00 & \\
\hline$p$ value & 0.694 & 0.497 & \\
\hline
\end{tabular}

PHQ-9, Patient Health Questionnaire-9; CORE-OM, Clinical Outcomes in Routine Evaluation - Outcome Measure; SF-36 MCS, Short Form Health Survey mental component score; SF-36 PCS, Short Form Health Survey physical component score.

Consequently the results have wide confidence intervals around the mean and the effect size we obtained requires confirmation in a full trial. Although we did control for baseline depression severity as a covariate in our analysis, small numbers also prevented us balancing potentially important variables such as ethnicity, marital status and gender in our randomization which may or may not have influenced our results. Further, although there were no differences in consultations with GPs between groups, we do not have full detailed information on what constituted usual care in the control groups, which may have varied substantially and affected our results. However, Phase II trials are an important stage in carefully developing and testing new interventions and these results lend support to the utility of the MRC's complex-interventions research framework (MRC, 2000), which provided a logical and systematic structure to help us in the process of designing and testing collaborative care in the UK. For example, during our development work, we found four previous UK studies of collaborative care which in contrast to our results produced no or inconclusive effects (Wilkinson et al. 1993; Blanchard et al. 1995; Mann et al. 1998; Peveler et al. 1999). These studies were early trials in the development of collaborative care, and had not used the systematic framework to develop their interventions; our review indicates they had used suboptimal intervention ingredients (Gilbody et al. 2006). Although the principle of carefully phased intervention development is an effective way to think about designing interventions and is supported by our results, the framework is not prescriptive and lacks close detail. Our specific approach is only one of many methodological possibilities.

The research implications are that a fully powered Phase III cluster-randomized trial should be the next step of the MRC's complex-interventions phased approach (Campbell et al. 2000; MRC, 2000) to investigating this complex intervention. Such a design will provide the best protection against both over- and underestimating the real effect size of collaborative care in the UK and will allow us to achieve a better balance of baseline demographic characteristics. A parallel qualitative investigation to this trial (Richards et al. 2006b) has shown the clinical procedures to be acceptable to patients, mental health workers and GPs. If such a trial were to confirm the effect size of our Phase II trial results, we will have evidence to enable the NHS to substantially improve the organization of its care for depressed patients in primary care and to assist primary-care providers to deliver an effective 
model of enhanced depression service within the GP contract.

\section{Acknowledgements}

This trial was funded by MRC grant no. G03000677; ID: 68073, International Standard RCT no.: ISRCT63222059. The researchers worked independently of the research funder.

\section{Declaration of Interest}

None.

\section{References}

Barkham M, Margison F, Leach C, Lucock M, Mellor-Clark J, Evans C, Benson, L, Connell J, Audin K, McGrath G (2001). Service profiling and outcomes benchmarking using the CORE-OM: towards practice-based evidence in the psychological therapies. Journal of Consulting and Clinical Psychology 69, 184-196.

Blanchard M, Waterreus A, Mann A (1995). The effect of primary care nurse intervention upon older people screened as depressed. International Journal of Geriatric Psychiatry 10, 289-298.

Bower P, Gilbody S (2005). Managing common mental health disorders in primary care: conceptual models and evidence base. British Medical Journal 330, 839-842.

Campbell M, Fitzpatrick R, Haines A, Kinmonth AL, Sandercock P, Spiegelhalter D, Tyrer P (2000). Framework for design and evaluation of complex interventions to improve health. British Medical Journal 321, 694-696.

Cohen J (1988). Statistical Power Analysis for the Behavioral Sciences. Erlbaum: Hillsdale, NJ.

Cuijpers P, van Straten A, Warmerdam L (2007). Behavioural activation treatments of depression: a meta analysis. Clinical Psychology Review 27, 318-326.

Dimidjian S, Hollon S, Dobson K, Schmaling KB, Kohlenberg RJ, Addis ME, Gallo R, McGlinchey JB, Markley DK, Gollan JK, Atkins DC, Dunne, DL, Jacobson NS (2006). Randomized trial of behavioural activation, cognitive therapy, and antidepressant medication in the acute treatment of adults with major depression. Journal of Consulting and Clinical Psychology 74, 658-670.

Gensichen J, Beyer M, Muth C, Gerlach FM, Von Korff M, Ormel J (2005). Case management to improve major depression in primary health care: a systematic review. Psychological Medicine 36, 7-14.

Gilbody S, Bower P, Fletcher J, Richards DA, Sutton A (2006). Collaborative care for depression: a systematic review and cumulative meta-analysis. Archives of Internal Medicine 166, 2314-2321.

Gilbody S, Whitty P, Grimshaw J, Thomas R (2003). Educational and organizational interventions to improve the management of depression in primary care: a systematic review. Journal of the American Medical Association 289, 3145-3151.
Gunn J, Diggens J, Hegarty K, Blashki G (2006). A systematic review of complex system interventions designed to increase recovery from depression in primary care. BMC Health Services Research 6, 88.

Jacobson N, Dobson K, Traux P, Addis ME, Koerner K, Gollan JK, Gortner E, Prince SE (1996). A component analysis of cognitive-behavioural treatment of depression. Journal of Consulting and Clinical Psychology 64, 295-304.

Katon W, Von Korff M, Lin E, Simon G, Walker E, Unützer J, Bush T, Russo J, Ludman E (1999). Stepped collaborative care for primary care patients with persistent symptoms of depression: a randomized trial. Archives of General Psychiatry 56, 1109-1115.

Killaspy H, Bebbington P, Blizard R, Johnson S, McCrone P, Nolan F, Pilling S, King M (2005). The REACT study: a Randomised Evaluation of Assertive Community Treatment in North London. British Medical Journal 331, 599-604.

Kroenke K, Spitzer RL, Williams JB (2001). The PHQ-9: validity of a brief depression severity measure. Journal of General Internal Medicine 16, 606-613.

Layard R (2006). The case for psychological treatment centres. British Medical Journal 332, 1030-1032.

Lipsey M, Wilson D (2001). Practical Meta-Analysis. Sage: New York.

Mann A, Blizard R, Murray J, Smith JA, Botega N, MacDonald E, Wilkinson G (1998). An evaluation of practice nurses working with general practitioners to treat people with depression. British Journal of General Practice $48,875-879$.

Martell CR, Addis ME, Jacobson NS (2001). Depression in Context: Strategies for Guided Action. Norton: New York.

MRC (2000). A Framework for Development and Evaluation of RCTs for Complex Interventions to Improve Health. Medical Research Council: London.

National Institute for Health and Clinical Excellence (2004). Depression: Management of Depression in Primary and Secondary Care (Clinical guideline 23). NICE: London.

Peveler R, George C, Kinmonth A, Campbell M, Thompson C (1999). Effect of antidepressant drug counselling and information leaflets on adherence to drug treatment in primary care: randomised controlled trial. British Medical Journal 319, 612-615.

Richards DA, Barkham M, Bower P, Gask L, Gilbody S, Lovell K, Rogers A, Torgerson D, Escott D, Fletcher J, Hennessy S, Kendall S, Lankshear AJ, Richardson R, Simpson A (2006b). A Trial Platform of Enhanced Care for Depression in Primary Care: Final Report. University of York: York.

Richards DA, Lankshear AJ, Fletcher J, Rogers A, Barkham M, Bower P, Gask L, Gilbody S, Lovell K (2006a). Developing a UK protocol for collaborative care: a qualitative study. General Hospital Psychiatry 28, 296-305.

Simon G (2006). Collaborative care for depression. British Medical Journal 332, 249-250.

Singleton N, Bumpstead R, O'Brien M, Lee A, Meltzer HY (2001). Office of National Statistics: Psychiatric Morbidity Among Adults Living in Private Households. HMSO: London. 
Spitzer RL, Williams JB, Gibbon M, First MB (1992). The Structured Clinical Interview for DSM-III-R (SCID). I: History, rationale, and description. Archives of General Psychiatry 49, 624-629.

Ukoumunne OC, Gulliford MC, Chinn S, Sterne JA, Burney PG, Donner A (1999). Methods in health service research. Evaluation of health interventions at area and organisation level. British Medical Journal 319, 376-379.

Unutzer J, Katon W, Callahan CM, Williams Jr. JW, Hunkeler E, Harpole L, Hoffing M, Della Penna RD, Noel PH, Lin EH, Arean PA, Hegel MT, Tang L, Belin TR, Oishi S, Langston C, IMPACT Investigators (2002). Collaborative care management of late-life depression in the primary care setting: a randomized controlled trial. Journal of the American Medical Association 288, 2836-2845.

Von Korff M, Goldberg D (2001). Improving outcomes in depression. British Medical Journal 323, 948-949.

Wagner EH, Austin BT \& Von Korff M (1996). Organizing care for patients with chronic illness. Milbank Quarterly 74, 511-544.
Ware JE, Kosinski M, Dewey JE (2000). How to Score Version Two of the SF-36 Health Survey. Quality Metric Incorporated: Lincoln, RI.

Wells KB, Sherbourne C, Schoenbaum M, Duan N, Meredith L, Unutzer J, Miranda J, Carney MF, Rubenstein LV (2000). Impact of disseminating quality improvement programs for depression in managed primary care: a randomized controlled trial. Journal of the American Medical Association 283, 212-220.

White H (1980). A heteroskedasticity-consistent covariance matrix estimator and a direct test for heteroskedasticity. Econometrica 48, 817-838.

Wilkinson G, Allen P, Marshall E, Walker J, Browne W, Mann AH (1993). The role of the practice nurse in the management of depression in general practice: treatment adherence to antidepressant medication. Psychological Medicine 23, 229-237.

WHO (2001). The World Health Report 2001: Mental Health: New Understanding, New Hope. World Health Organisation: Geneva. 